\title{
The Variability of the Order Burkholderiales Representatives in the Healthcare Units
}

\author{
Olga L. Voronina, ${ }^{1}$ Marina S. Kunda, ${ }^{1}$ Natalia N. Ryzhova, ${ }^{1}$ Ekaterina I. Aksenova, ${ }^{1}$ \\ Andrey N. Semenov, ${ }^{1}$ Anna V. Lasareva, ${ }^{2}$ Elena L. Amelina, ${ }^{3}$ Alexandr G. Chuchalin, ${ }^{3}$ \\ Vladimir G. Lunin, ${ }^{1}$ and Alexandr L. Gintsburg ${ }^{1}$ \\ ${ }^{1}$ N.F. Gamaleya Federal Research Center for Epidemiology and Microbiology, Ministry of Health of Russia, Gamaleya Street 18, \\ 123098 Moscow, Russia \\ ${ }^{2}$ Federal State Budgetary Institution "Scientific Centre of Children Health" RAMS, 119991 Moscow, Russia \\ ${ }^{3}$ Research Institute of Pulmonology FMBA of Russia, 105077 Moscow, Russia
}

Correspondence should be addressed to Olga L. Voronina; kirolg3@newmail.ru

Received 12 September 2014; Accepted 1 December 2014

Academic Editor: Vassily Lyubetsky

Copyright (C) 2015 Olga L. Voronina et al. This is an open access article distributed under the Creative Commons Attribution License, which permits unrestricted use, distribution, and reproduction in any medium, provided the original work is properly cited.

\begin{abstract}
Background and Aim. The order Burkholderiales became more abundant in the healthcare units since the late 1970s; it is especially dangerous for intensive care unit patients and patients with chronic lung diseases. The goal of this investigation was to reveal the real variability of the order Burkholderiales representatives and to estimate their phylogenetic relationships. Methods. 16S rDNA and genes of the Burkholderia cenocepacia complex (Bcc) Multi Locus Sequence Typing (MLST) scheme were used for the bacteria detection. Results. A huge diversity of genome size and organization was revealed in the order Burkholderiales that may prove the adaptability of this taxon's representatives. The following variability of the Burkholderiales in Russian healthcare units has been revealed: Burkholderiaceae (Burkholderia, Pandoraea, and Lautropia), Alcaligenaceae (Achromobacter), and Comamonadaceae (Variovorax). The Burkholderia genus was the most diverse and was represented by 5 species and 16 sequence types (ST). ST709 and 728 were transmissible and often encountered in cystic fibrosis patients and in hospitals. A. xylosoxidans was estimated by 15 genotypes. The strains of first and second ones were the most numerous. Conclusions. Phylogenetic position of the genus Lautropia with smaller genome is ambiguous. The Bcc MLST scheme is applicable for all Burkholderiales representatives for resolving the epidemiological problems.
\end{abstract}

\section{Introduction}

The antibiotic era resulted in the cardinal changes in the spectrum of the microorganisms, causing the healthcareassociated infections. Well-known bacterial pathogen Staphylococcus aureus was crowded by Pseudomonas aeruginosa [1]; then both were pressed by other Proteobacteria. The resistome of these bacteria has been enriched over the years of the nosocomial circulation, but most of them kept sensitive to at least one antibiotic.

The situation was complicated by the appearance of the order Burkholderiales bacteria after the late 1970s [2]. These bacteria are the common inhabitants of soil and water. They can be the plants' pathogens and have natural resistance to common antibiotics. They are especially dangerous for intensive care unit patients and patients with chronic lung diseases, particularly cystic fibrosis [3]. The taxonomy of this bacteria group has been developing since the 1980s and they were subdivided into different genera between 1981 and 2000 [4-8].

The infusion of nucleic acid sequencing technology in microbiology allowed Woese to start solving the bacterial phylogeny problem [9]. Proteobacteria, the most abundant and diverse bacterial phyla, were subdivided into classes on the base of the 16S rRNA gene sequences. First of all, 8-12 nucleotide signature sequences whose characteristic is unique to the species of Beta- and Gammaproteobacteria were identified (AAAAACCUUACC for Betaproteobacteria; AAACUCAAAUG for Gammaproteobacteria) [10, 11]. So the taxon Pseudomonas, according to Woese, was actually 
a collection of at least five separate groups of bacteria [9]. It was subdivided into several genera, one of which was genus Burkholderia [5]. Later a new genus Ralstonia was separated from Burkholderia [7]. Lautropia [6] and Pandoraea [8] have appeared in the last few years. However the diversity, clinical and epidemiological significance of these taxa bacteria needs in detailed study. Continuing the investigation of Bcc role in nosocomial infections and using Multilocus Sequence Typing (MLST) as successful methodology in the epidemiology [12], we attempted to understand the variability of Burkholderiales in healthcare units.

\section{Materials and Methods}

2.1. Materials. Biological samples used for sequencing data are divided into two parts. The first part predominantly represented by nosocomial strains and strains from cystic fibrosis (CF) patients was described in [13] in detail. The second part contained some strains and mainly specimens of human sputum and aspirates from more than $300 \mathrm{CF}$ patients.

2.2. DNA Isolation. DNA for PCR analysis was extracted from the bacterial cultures as described previously [13]. DNA from sputum and aspirate was isolated according to the protocol of the Maxwell 16 Tissue DNA Purification Kit for Maxwell MDX Instrument (Promega).

2.3. Species Identification. Identification of species was performed by amplification and sequencing of $16 \mathrm{~S}$ ribosomal RNA gene (16S rDNA) fragment with primers $[14,15]$.

2.4. MLST. For Multilocus Sequence Typing, a modified scheme that allows differentiating 19 species of the Burkholderia cepacia complex $(B c c)$ was used [16]. The scheme includes the following targets for amplification: atpD, a $\beta$ chain of ATP synthase; $g l t B$, a large subunit of glutamate synthase; $g y r B$, a $B$ subunit of DNA gyrase; $r e c A$, recombinase A; lepA, a GTP binding protein; phaC, acetyl CoA reductase; and $\operatorname{trp} B$, a $\mathrm{B}$ subunit of tryptophane synthase. For DNA amplification, the following reagents were used: hot rescue DNA pol 5 units/ $\mu \mathrm{L}$, PCR buffer 10x (N.F. Gamaleya Institute for Epidemiology and Microbiology MoH), dNTP5 mM (Medigen), and primers (Evrogen). The modified amplification program was the same for all targets: $95^{\circ} \mathrm{C}-10 \mathrm{~min}\left(95^{\circ} \mathrm{C}-30 \mathrm{~s}, 63^{\circ} \mathrm{C}-\right.$ $\left.40 \mathrm{~s}, 72^{\circ} \mathrm{C}-1 \mathrm{~min}\right) \times 35,72^{\circ} \mathrm{C}-5 \mathrm{~min}$.

2.5. PCR Products Sequencing. PCR products were sequenced according to the protocol of BigDye Terminator 3.1 Cycle Sequencing kit for the Genetic Analyzer 3130 of Applied Biosystems/Hitachi. The electrophoretic DNA separation was performed in $50 \mathrm{~cm}$ capillaries with POP7 polymer.

2.6. Nucleotide Sequence Analysis. Analysis of sequences and alignment were made by the use of the program ClustalW2 [17]. Allele numbers for MLST genes were assigned with the help of the PubMLST website [18]. New alleles and STs were controlled and submitted by the curator of Bcc MLST database. Identification of $16 S$ rDNA sequences was carried out by BLAST search.
2.7. Nucleotide Sequence Polymorphism. The numbers of nucleotide/amino acid differences per site between concatenated sequences of $17 \mathrm{Bcc}$ STs were obtained by pairwise distance calculation. Analyses were conducted in MEGA 4.0 [19].

Percent similarity and divergence coefficients of $g l t B$ gene nucleotide/amino acid sequences among analyzed representatives of the Burkholderiales were performed by the use of ClustalW2 [17], MEGA 6.0 [20], and MegAlign 5.05. For comparative sequence analysis and phylogenetic reconstruction 10 extra gltB gene sequences of the Burkholderiales order representatives (Ralstonia solanacearum, Ralstonia pickettii, Acidovorax citrulli, Variovorax paradoxus, Bordetella bronchiseptica, Bordetella pertussis, and Lautropia mirabilis) were retrieved from GenBank database (Table 3). The gltB sequences of Pseudomonas aeruginosa have been used as outgroup taxon (Table 3 ).

2.8. Phylogenetic Analysis. Phylogenetic analysis of Bcc was performed based on allelic profile data of Bcc STs and translated concatenated sequences of seven MLST loci. Phylogenetic tree of analyzed representatives of Burkholderiales order was constructed by the use of $g l t B$ sequences.

Analysis of profile data of Bcc STs was conducted using the software packages SplitsTree [21].

The phylogenetic tree of $17 \mathrm{Bcc}$ STs based on translated concatenated sequences of seven MLST loci was obtained automatically by applying the neighbor-joining method [22]. The evolutionary distances between $17 \mathrm{Bcc}$ STs were computed using the $p$-distance method [23] and were evaluated through the units of the amino acid differences' number per site. Evolutionary analyses were conducted in MEGA 6.0 [20]. Bootstrap analyses were performed with 500 replicates.

Phylogenetic tree of analyzed representatives of the Burkholderiales order was constructed by the use of neighborhood-joining, maximum likelihood, and maximum parsimony methods.

Genetic distances between microorganisms were evaluated by the use of Tamura 3-parameter model [24], which was chosen as an optimal evolution distance model derived from Modeltest based on the Akaike information criterion [25]. The evolutionary history was inferred by using the maximum likelihood method based on the general time reversible model GTR+G. Initial trees for the heuristic search were obtained automatically by applying neighbor-joining and BioNJ algorithms to a matrix of pairwise distances estimated by the use of the maximum composite likelihood approach and then selecting the topology with superior log likelihood value. A discrete gamma distribution was used to model evolutionary rate differences among sites $(+\mathrm{G}$, parameter $=$ $0.8170)$. Maximum parsimony trees were constructed with an algorithm implemented in MEGA 6.0. Bootstrap analyses were performed with 1,000 replicates.

\section{Results and Discussion}

3.1. Common Characteristics of the Burkholderiales Genomes. The Burkholderiales is the dominating order among the 
TABLE 1: The representatives of four families of the order Burkholderiales, which were detected in clinical specimens.

\begin{tabular}{|c|c|c|c|c|}
\hline Class & \multicolumn{4}{|c|}{ Betaproteobacteria } \\
\hline Order & \multicolumn{4}{|c|}{ Burkholderiales } \\
\hline Family & Comamonadaceae & Alcaligenaceae & Burkholderiaceae & Ralstoniaceae \\
\hline Genus & Acidovorax & Bordetella & Burkholderia & Ralstonia \\
\hline Genus & Variovorax & Achromobacter & Pandoraea & \\
\hline & & & Lautropia & \\
\hline
\end{tabular}

$\beta$-Proteobacteria available genomes, covering six families: Alcaligenaceae, Burkholderiaceae, Comamonadaceae, Oxalobacteraceae, Ralstoniaceae, and Sutterellaceae [26]. Four of them, demonstrated in Table 1, are more vital for the healthcare units. In the context of genome size, the order Burkholderiales is extraordinarily various (see S1 in Supplementary Material available online at http://dx.doi.org/ $10.1155 / 2014 / 680210$ ): from the smallest $0.070281 \mathrm{Mb}$ of the Burkholderiales bacterium JGI 0001003-L21 (the rhizosphere and endosphere of Arabidopsis thaliana, INSDC AUNS00000000.1 [27]) to the biggest $11.2941 \mathrm{Mb}$ of the Burkholderia terrae (the forest soil, INSDC AKAU00000000.1 [28]). The genomes of the Burkholderiales representatives are organized in different number of the chromosomes: 1,2 , or 3 , without genome size correlation. So $7.35915 \mathrm{Mb}$ genome of Achromobacter xylosoxidans has one chromosome, but $7.00881 \mathrm{Mb}$ genome of Burkholderia multivorans has three chromosomes [27].

Analysis of the small genome group has demonstrated that all of them are the genomes of the host-restricted microbial symbionts: of plants, as abovementioned Burkholderiales bacterium JGI 0001003-L21 (INCDS AUNS00000000.1, genome size $0.07 \mathrm{Mb}$ ) [27], of sap-feeding insects, as Candidatus Zinderia insecticola (INCDS CP002161.1, genome size $0.208564 \mathrm{Mb}$ ) [29], or of human, as Burkholderiales bacterium1_1_47 (INCDS ADCQ00000000.1, genome size 2.61 Mb), isolated from feces in Human Microbiome Project [27].

But most of the bacteria of Burkholderia cepacia complex $(B c c)$ pathogenic for human keep big genome, providing for the genome plasticity and adaptability [30].

3.2. Bcc Diversity in the Healthcare Units of the Russian Federation. In our investigation of the microorganisms, causing the healthcare-associated infections, we drew attention to $B c c$ bacteria in departments both common and specialized for cystic fibrosis (CF) patients. Thirteen genotypes (sequence type, ST) were detected in the first phase of the analysis and nine of them $(708,709,710,711,712,714,727,728$, and 729) were identified for the first time (Table 2). It was shown that strains causing nosocomial infections in most cases refer to genotypes 728 and 708. Genotype 709 detected in strains isolated from patients in seven federal regions of Russia should be recognized as epidemiologically significant for patients with cystic fibrosis [13].

The extension of the specimens' sampling in the second phase of the investigation demonstrated new $B c c$ genotypes in B. cenocepacia (ST862, 878) and B. multivorans species (ST835) and continued prevalence of the ST709. 79\% of

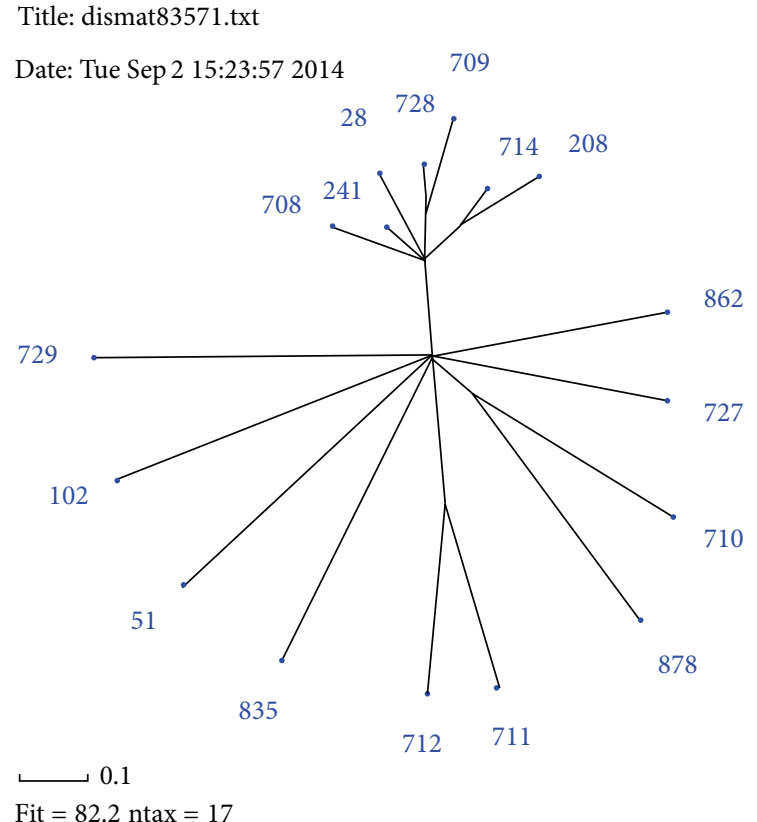

FIGURE 1: SplitsTree on the base of allelic profile data of the Burkholderia cepacia complex STs from RF.

the CF patients, infected by Bcc, had ST709 strain. So, 16 STs were detected for $B c c$ isolated from patients in RF.

To establish the relationship between different STs, we applied several methods of analysis. The first of them was SplitsTree analysis which was performed on the base of allelic profile data of Bcc STs (Figure 1). The most numerous group was formed by 6 STs of B. cenocepacia $(708,241,728,709$, 714 , and 208) closely related to the globally spread ST28. Next small groups were two STs containing first STs 710 and 878 that belonged to B. cenocepacia and second STs 711 and 712 related to $B$. multivorans. The other STs formed the separate branches.

To estimate the changes in the amino acid sequences, the concatenated sequences of MLST loci were translated. The bootstrap consensus tree using the neighbor-joining method was created (Figure 2). All groups of STs, represented different Burkholderia species, formed the separate branches with high bootstrap index (BI).

The most numerous group was formed by B. cenocepacia. It included 11 STs. Despite the fact that ST241, ST28, ST728, and ST709 had double locus variation (DLV) in the allelic profile, they formed the same branch with BI 63\%. ST 708 which is also the DLV from ST241 was away from the group. 
TABLE 2: The characteristics of the $B c c$ genotypes identified in RF.

\begin{tabular}{|c|c|c|c|c|c|}
\hline Species & ST & ID PubMLST & Year & Source & Comments for ST distribution \\
\hline B. cenocepacia & 708 & 1149 & 2001 & NON & Nosocomial strains in RF \\
\hline B. cenocepacia & 241 & 1258 & 2012 & $\mathrm{CF}$ & $\begin{array}{l}\text { Only CF strain in Far East of RF, but intercontinental spread strain in the } \\
\text { world }\end{array}$ \\
\hline B. cenocepacia & 28 & 1268 & 1989 & $\mathrm{CF}$ & $\begin{array}{l}\text { Strains of multiple globally distinct locations, except for RF. Reference strain } \\
\text { from Belgian collection }\end{array}$ \\
\hline B. cenocepacia & 728 & 1248 & 2004 & $\mathrm{NON}$ & Nosocomial epidemic strains in RF, CF strain in all federal regions of RF \\
\hline B. cenocepacia & 709 & 1150 & 2008 & $\mathrm{CF}$ & Epidemic strains for CF patients in all federal regions of RF, except for Far East \\
\hline B. cenocepacia & 714 & 1155 & 2003 & NON & Strain from one hospital of the Southern Federal Region of RF \\
\hline B. cenocepacia & 208 & 1261 & 2012 & $\mathrm{CF}$ & CF strains in Southern and Volga Federal Regions of RF and in USA \\
\hline B. cenocepacia & 862 & 1466 & 2014 & $\mathrm{CF}$ & CF strain only in Far East of RF \\
\hline B. cenocepacia & 727 & 1246 & 2002 & NON & Nosocomial strains in Northwestern Federal Region of RF \\
\hline B. cenocepacia & 710 & 1151 & 2012 & $\mathrm{CF}$ & CF strains in RF \\
\hline B. cenocepacia & 878 & 1501 & 2014 & $\mathrm{CF}$ & CF strain in RF \\
\hline B. multivorans & 711 & 1152 & 2012 & $\mathrm{CF}$ & CF strains in RF \\
\hline B. multivorans & 712 & 1153 & 2011 & CF & CF strains in RF and in Spain \\
\hline B. multivorans & 835 & 1443 & 2013 & CF & CF strain in RF \\
\hline B. stabilis & 51 & 1267 & 1998 & $\mathrm{NON}$ & $\begin{array}{l}\text { Nosocomial strain in one hospital of RF, but intercontinental spread strain in } \\
\text { the world }\end{array}$ \\
\hline B. contaminans & 102 & 1264 & 2000 & $\mathrm{CF}$ & $\begin{array}{l}\text { Nosocomial strain in one hospital and CF strain in Northwestern Federal } \\
\text { Region of RF, but intercontinental spread strain in the world }\end{array}$ \\
\hline B. vietnamiensis & 729 & 1266 & 2012 & $\mathrm{CF}$ & CF strain only in Far East of RF \\
\hline
\end{tabular}

CF: cystic fibrosis patient; NON: non-CF patient; RF: Russian Federation.

This can be explained by amino acid residues changes in the translated MLST sequences. In fact, between ST28, ST241, ST709, and ST728 there were no amino acid residues replacements in translated sequences, while ST708 had replacement V82A in large subunit of glutamate synthase. STs 714 and 208 with DLV in their allelic profiles formed a separate subgroup $(\mathrm{BI}=100 \%)$. These STs differed from the other STs with the replacement E52D in ATP synthase beta chain; ST208 had additional replacement E7D in acetoacetyl-CoA reductase.

Another subgroup of B. cenocepacia with $\mathrm{BI}=79 \%$ was represented by strains with STs 727, 862, 710, and 878 .

Evolutionary divergence between sequences of 17 STs was received by pairwise distance calculation (Supplementary Material, S2 and S3). The less variability (0.002-0.005) was within the group including ST28, ST241, ST728, ST709, ST714, ST208, and ST708 related to B. cenocepacia (group 1). Group 2 was formed by B. cenocepacia strains too (ST727, ST862, ST710, and ST878). They had 0.019-0.023 base differences per site in comparison with group 1 . So, in whole intraspecies $B$. cenocepacia STs variability was $0.002-0.023$. The $B$. multivorans STs variability was almost the same$0.003-0.011$. The most closely related species in the analyzed sample of Bcc were B. cenocepacia and B. contaminans (ST102) with variability from 0.037 to 0.040 .

However, in most cases nucleotide rearrangement did not lead to changes in amino acid residues sequences and polymorphism within amino acid sequences was less than within nucleotides (Supplementary Material, S2 and S3). ST28, ST241, ST728, and ST709 had the same amino acid residues sequences. ST714, ST208, and ST708 differed from them with 0.001-0.003 amino acid residues per site. So,

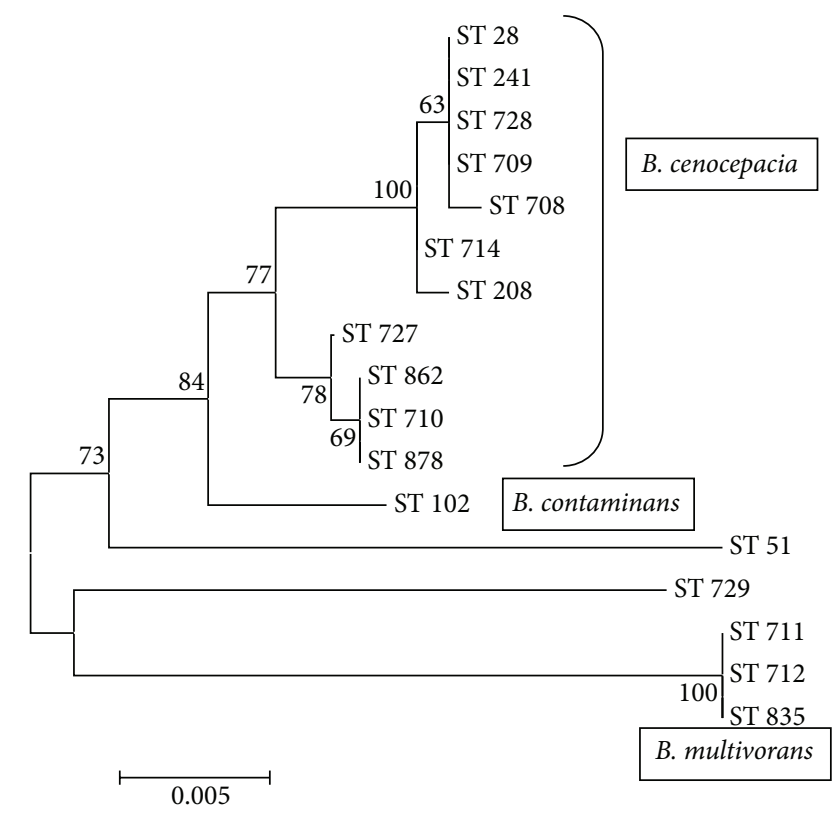

FIGURE 2: Neighbor-joining phylogenetic tree of seventeen Burkholderia cepacia complex STs based on translated concatenated sequences of seven MLST loci. ST51: B. stabilis; ST729: B. vietnamiensis.

the number of amino acid residues' differences per site in group 1 was $0.000-0.003$. More detectable changes were between STs group 1 and strains from group 2 (ST727, ST862, ST710, and ST878), 0.007-0.010 amino acid residues per site. 
TABLE 3: The sources of the gltB gene sequences for the phylogenetic analysis; ${ }^{*}$ consecutive laboratory numeration of the registered alleles.

\begin{tabular}{|c|c|c|c|}
\hline$N$ & Species & $\begin{array}{l}\text { Source or GenBank } \\
\text { accession number }\end{array}$ & $\begin{array}{c}\text { gltB allele or locus } \\
\text { tag }\end{array}$ \\
\hline 1 & B. cenocepacia & {$[18]$} & 11 \\
\hline 2 & B. cenocepacia & {$[18]$} & 16 \\
\hline 3 & B. stabilis & {$[18]$} & 18 \\
\hline 4 & B. multivorans & {$[18]$} & 50 \\
\hline 5 & B. multivorans & {$[18]$} & 60 \\
\hline 6 & B. contaminans & {$[18]$} & 80 \\
\hline 7 & B. vietnamiensis & {$[18]$} & 103 \\
\hline 8 & B. cenocepacia & {$[18]$} & 134 \\
\hline 9 & B. cenocepacia & {$[18]$} & 136 \\
\hline 10 & B. cenocepacia & {$[18]$} & 176 \\
\hline 11 & B. cenocepacia & {$[18]$} & 352 \\
\hline 12 & B. multivorans & {$[18]$} & 358 \\
\hline 13 & A. xylosoxidans & KC817498 & $1^{*}$ \\
\hline 14 & A. xylosoxidans & KC817500 & $2^{*}$ \\
\hline 15 & A. xylosoxidans & KF290958 & $3^{*}$ \\
\hline 16 & A. xylosoxidans & KF290959 & $4^{*}$ \\
\hline 17 & A. xylosoxidans & KJ941209 & $5^{*}$ \\
\hline 18 & A. xylosoxidans & KF297891 & $6^{*}$ \\
\hline 19 & A. xylosoxidans & KF963246 & $7^{*}$ \\
\hline 20 & A. xylosoxidans & KF963247 & $8^{*}$ \\
\hline 21 & A. xylosoxidans & KF963248 & $9^{*}$ \\
\hline 22 & A. xylosoxidans & KF963249 & $10^{*}$ \\
\hline 23 & A. xylosoxidans & KF963250 & $11^{*}$ \\
\hline 24 & A. xylosoxidans & KJ364657 & $12^{*}$ \\
\hline 25 & A. xylosoxidans & KJ439616 & $13^{*}$ \\
\hline 26 & A. xylosoxidans & KM262752 & $14^{*}$ \\
\hline 27 & A. xylosoxidans & KM262753 & $15^{*}$ \\
\hline 28 & R. solanacearum & AL646052.1 & RSc2965 \\
\hline 29 & R. pickettii & СР006667.1 & N234_19250 \\
\hline 30 & A. citrulli & СР000512.1 & Aave_1008 \\
\hline 31 & V. paradoxus & СР001635.1 & Vapar_1152 \\
\hline 32 & B. bronchiseptica & HE965806.1 & BN112_3590 \\
\hline 33 & B. pertussis & BX640422.1 & BP3753 \\
\hline 34 & P. pnomenusa & KM410934 & KM410934 \\
\hline 35 & P. pnomenusa & СР007506.1 & DA70_18115 \\
\hline 36 & L. mirabilis & KM410932 & KM410932 \\
\hline 37 & L. mirabilis & KM410933 & KM410933 \\
\hline 38 & L. mirabilis & AEQP01000020.1 & EFV94423.1 \\
\hline 39 & P. aeruginosa & AE004091.2 & PA5036 \\
\hline 40 & P. aeruginosa & FM209186.1 & PLES_54261 \\
\hline
\end{tabular}

So, B. cenocepacia ST241, ST728, ST709, ST714, ST208, and ST708 formed clonal complex, including ST28, which characterized the strains with global spread. Most of these STs were typical only for RF healthcare units (Table 2). Three STs $(728,708$, and 709$)$ were adaptive for epidemic spread.
3.3. The Potential of the Bcc MLST Scheme in the Burkholderiales Representatives Detection. During the second phase of the investigation we dealt not only with bacterial strains but also with a lot of samples of the sputum and aspirate. The Bcc MLST scheme adaptation to new conditions, amplification Bcc DNA in total DNA of the sample, suggested the apprehension of Spilker et al. [16] that degenerate primers, which allowed expansion of the modified Bcc MLST scheme, would not be specific only for Burkholderia species. First representative of Burkholderiales was Achromobacter xylosoxidans, in which gltB gene was amplified with the $B c c$ MLST scheme primers. After including this sequence in the analysis of the samples, we identified two different $g l t B$ alleles for this bacterium from the CF patients [13]. Then another thirteen gltB alleles were detected for $A$. xylosoxidans. The data analysis demonstrated the prevalence of the allele 1 and allele 2 among the CF patients from all federal regions of RF, except Far East, where only allele 3 was registered for $A$. xylosoxidans.

The increase of the number of $A$. xylosoxidans cases in the healthcare units, not only in CF patients, is according to the data of the new species registration. The data analysis of List of Prokaryotic Names with Standing in Nomenclature for the Burkholderiales order members demonstrated that over the last two years 18 new species of the Burkholderia genus have been registered [31], but only one was isolated from the human respiratory sample and others were environmental. On the other hand, species number of the Achromobacter genus increased two times during this period. All eight new species were clinical [32]. This data suggested Achromobacter significance as nosocomial bacterium.

Two targets of Pandoraea pnomenusa (recA and gltB) were amplified with the primers of Bcc MLST scheme too. But this dangerous and transmissible bacterium was isolated only from one CF patient. Some cases of Lautropia mirabilis were registered within one period of time. Only gltB gene was detectable by Bcc MLST primers. At last Variovorax paradoxus, detected in the group of the patients' samples, was amplified with gyrB primers. Detection of the seldom trace amount of Ralstonia spp. was possible with the gltB primers too.

So, we may conclude that the Bcc MLST scheme gltB primers are universal for most of the clinically significant Burkholderiales. The gltB gene sequences from our investigation and sequences avoided from GenBank were analyzed in the next step. Bordetella genus sequence included was explained by the importance of this genus as causative agent of human diseases.

3.4. The gltB Gene Sequence Polymorphism. 38 representatives of the order Burkholderiales and two of Pseudomonas aeruginosa (as an outgroup taxon) were used in analysis. A 414-base-pair alignment for the gltB gene region was obtained.

Totally 295 variable nucleotide sites have been detected; 240 of them characterized diversity of the orderBurkholderiales analyzed representatives. Differences between representatives of the Burkholderiales and the outgroup taxa, Pseudomonas aeruginosa, reached 48.8\% (Pse-Ral, 
Pse-Var-Aci); see Table 4. The differences among the investigated Burkholderiales bacteria varied from $0.2 \%$ to $32.5 \%$. The gltB allele diversity in $B c c$ represented in analysis by five species was comparable to the diversity among $A$. xylosoxidans alleles and reached $6.8 \%$ and $5.1 \%$, respectively (Supplementary Material, S4). These data suggested the close relatedness of the $B c c$ species. Percent of the differences in glt $B$ gene sequence between representatives of the Alcaligenaceae family, A. xylosoxidans and Bordetella bronchiseptica/Bordetella pertussis, was $6.8-9.2 \%$, indicating close relatedness of these taxa too. The gltB allele differences between other representatives of Burkholderiales fell into the range of $20-32.5 \%$.

Surprisingly, the level of gltB gene sequence differences between the members of one family Burkholderiaceae (Bcc and Lautropia mirabilis) reached 27.9-31.6\%, and that was more than differences between $B c c$ and the member of the other families: Ralstoniaceae (Ralstonia, 20.6-26.9\%) and Comamonadaceae (Acidovorax, Variovorax, 25.2-28.6\%) (Supplementary Material, S4). However, according to the data of Gerner-Smidt, based on variability of $16 S$ rDNA the differences between Lautropia mirabilis and Burkholderia cepacia were $7.7 \%$ [6], which can characterize the higher resolution features of $g l t B$ gene sequences.

Amino acid residues variability of the translated gltB gene fragments was also evaluated (Supplementary Material, S5). In the sequence, consisting of 136 amino acid residues, 111 residues were variable. So, out of 240 SNPs, characterizing the diversity of Burkholderiales, 81 SNPs resulted in amino acid residues substitutions. The interspecies diversity of $A$. xylosoxidans was characterized by six amino acid residues substitutions; the diversity of $B c c$, by nine substitutions.

3.5. Phylogeny of the Analyzed Burkholderiales Representatives Based on gltB Sequences. ML phylogenetic tree based on gltB sequence is presented in Figure 3. P. aeruginosa well known as nosocomial bacterial pathogen, taken in this analysis as an outgroup taxon (Gammaproteobacteria, Pseudomonadales, and Pseudomonadaceae), formed the most divergent basal branch on the tree as was expected. The phylogenetic tree revealed two main groups of the Burkholderiales order representatives in this analysis. The first group (BI 78\%) included only the members of the Alcaligenaceae family: fifteen alleles of $A$. xylosoxidans and reference gltB alleles from Bordetella genomes. It should be noted that gltB sequences allowed separating these taxa into distinct subclades.

The second group (BI 78\%) was formed by the representatives of three families: Burkholderiaceae, Ralstoniaceae, and Comamonadaceae. Inside the second group twelve alleles of $B c c$ formed a large subgroup; two alleles of Pandoraea pnomenusa were closely related to this subgroup. However, three alleles of Lautropia mirabilis were more divergent from gltB alleles of Bcc and Pandoraea pnomenusa than representatives of two different families Ralstoniacea (Ralstonia solanacearum, Ralstonia pickettii) and Alcaligenacea (Acidovorax citrulli, Variovorax paradoxus).

A similar situation was described by phylogenetic cladogram, constructed for the order Burkholderiales representatives [33] by an automated pipeline of PATRIC genome
TABle 4: Percent of gltB sequences variability among analyzed representatives of Burkholderiales.

\begin{tabular}{|c|c|c|}
\hline \multirow{2}{*}{$\begin{array}{l}\text { Group of } \\
\text { genotypes }\end{array}$} & \multicolumn{2}{|c|}{ Variability, \% } \\
\hline & DNA sequence & $\begin{array}{c}\text { Amino acid residues } \\
\text { sequence }\end{array}$ \\
\hline Lau & $4.1-4.4$ & $0.0-0.7$ \\
\hline Ach & $0.2-5.1$ & $0.0-3.7$ \\
\hline $\mathrm{Bcc}$ & $0.2-6.8$ & $0.0-5.1$ \\
\hline Ach-Bor & $6.8-9.2$ & $5.9-8.1$ \\
\hline Bcc-Pan & $21.1-23.1$ & $24.3-25.7$ \\
\hline Bcc-Bor & $25.5-26$ & $33.1-36$ \\
\hline Bcc-Ach & $23.8-26.7$ & $30.9-35.3$ \\
\hline Bcc-Ral & $20.6-26.9$ & $25-30.9$ \\
\hline Ach-Pan & $25.5-27.2$ & $25-27.9$ \\
\hline Bor-Pan & $26.9-27.4$ & $27.9-29.4$ \\
\hline Ral-Pan & $20.9-27.7$ & $24.3-27.2$ \\
\hline Bcc-Var-Aci & $25.2-28.6$ & $29.4-38.2$ \\
\hline Var-Pan & $28.2-29.1$ & $33.1-34.6$ \\
\hline Bor-Lau & $27.7-29.4$ & $32.4-33.1$ \\
\hline Var-Bor & $27.9-29.4$ & $35.3-39.7$ \\
\hline Ral-Var & $25.2-29.6$ & $36-38.2$ \\
\hline Ach-Var & $27.4-29.6$ & $35.3-41.9$ \\
\hline Ach-Lau & $28.2-30.3$ & $32.4-35.3$ \\
\hline Pan-Lau & $30.1-30.6$ & $30.9-31.6$ \\
\hline Ral-Bor & $24.5-31.3$ & $30.9-36.8$ \\
\hline Var-Lau & $29.4-31.3$ & $33.1-36$ \\
\hline Ach-Ral & $24-31.6$ & $28.7-38.2$ \\
\hline Bcc-Lau & $27.9-31.6$ & $30.1-33.1$ \\
\hline Ral-Lau & $26-32.5$ & $30.9-34.6$ \\
\hline Pse-Bor & $42.7-43$ & $66.2-66.9$ \\
\hline Ach-Pse & $43.7-45.9$ & $66.2-66.9$ \\
\hline Pse-Lau & $46.1-46.8$ & $69.1-69.9$ \\
\hline Bcc-Pse & $44.7-47.1$ & $65.4-66.2$ \\
\hline Pse-Pan & $47.6-47.8$ & 66.2 \\
\hline Pse-Ral & $46.1-48.8$ & $66.9-69.9$ \\
\hline Pse-Var & $47.3-48.8$ & $66.2-67.6$ \\
\hline
\end{tabular}

Bcc: Burkholderia cepacia complex; Ach: Achromobacter xylosoxidans; Lau: Lautropia mirabilis; Ral: Ralstonia solanacearum/Ralstonia pickettii; Aci: Acidovorax citrulli; Var: Variovorax paradoxus, Bor: Bordetella bronchiseptica/Bordetella pertussis; Pan: Pandoraea pnomenusa; Pse: Pseudomonas aeruginosa.

database [34]. The construction of the phylogenetic tree on this server begins with amino acid sequence files for each genome. On this tree Lautropia mirabilis fell in one group (BI 79\%) with two genomes of the hostrestricted microbial symbionts: Candidatus Zinderia insecticola (INCDS CP002161.1) [29] and Burkholderiales bacterium 1_1_47 (INCDS ADCQ00000000.1) [27], and with the representatives of the genera Parasutterella and Sutterella, the member of the family Sutterellaceae. Parasutterella was isolated from human faeces [35], and Sutterella strains were 


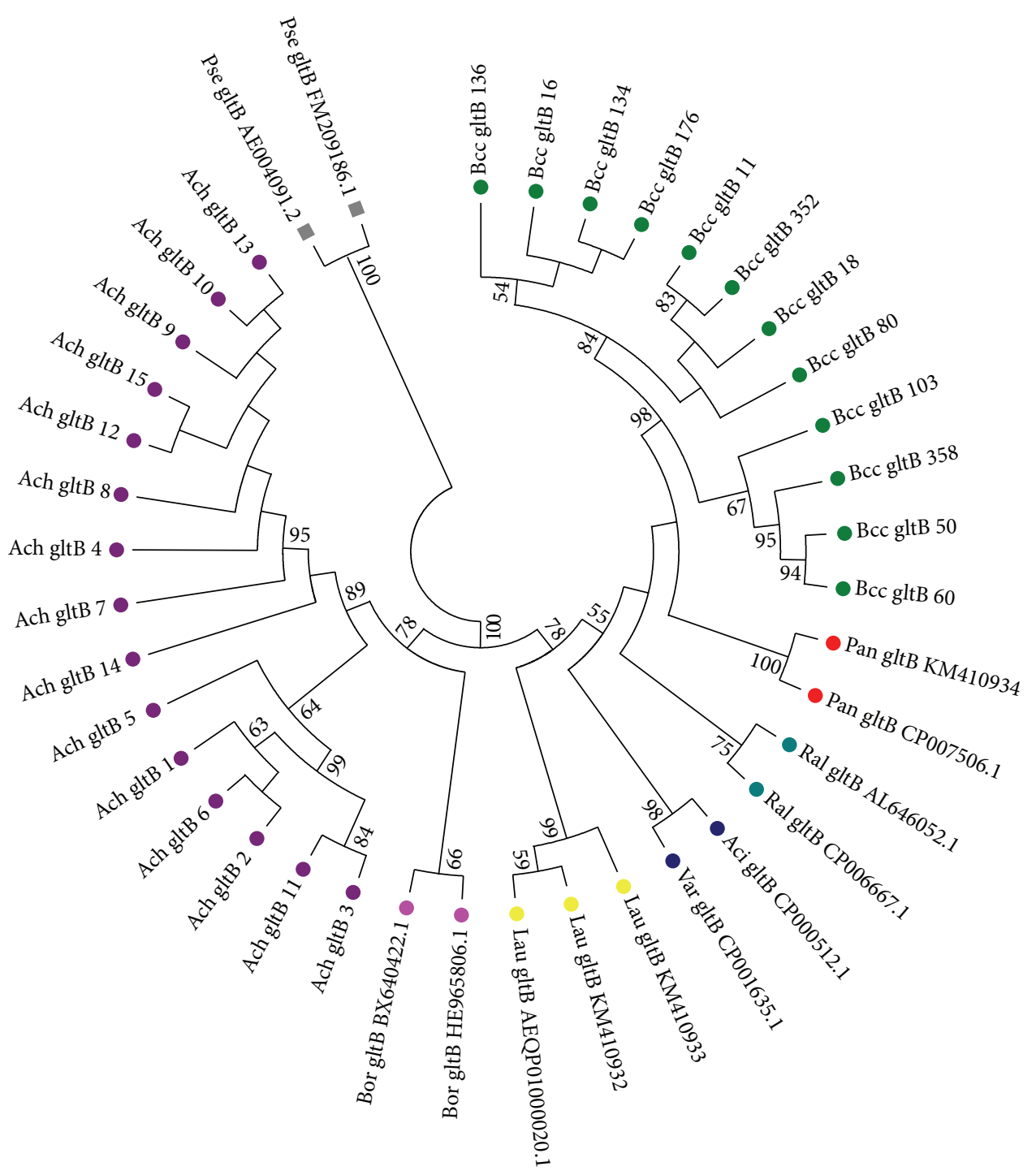

FIGURE 3: ML phylogenetic tree of analyzed representatives of Burkholderiales order based on gltB sequences.

isolated from infections that occurred below the diaphragm [36]. Both have small genome 2.3769-2.98833 Mb.

Lautropia mirabilis is the poorly investigated species of a Gram-negative motile coccus with the unusual morphology fairly recently isolated from the human mouth by GernerSmidt et al. [6]. The genome of this bacterium is surprisingly small ( $3.15192 \mathrm{Mb})$ as compared with $B c c$ and Pandoraea. The loss of some genes in the evolution of this bacterium may be suggested.

On the other hand, the 16S rDNA sequencing data showed that Lautropia mirabilis belonged to a separate branch of the Betaproteobacteria and was most closely related to the genus Burkholderia. The isolated position together with the unique combination of chemotaxonomic and phenotypic properties allowed attributing of Lautropia mirabilis (strain AB2188) to the separate genus [6].

According to the last All-Species Living Tree (Release LTPs115, March 2014) [37] and also based on 165 rDNA sequences, single Lautropia mirabilis (AEQP01000026) formed a separate basal branch more related to Burkholderia and Pandoraea in Burkholderiaceae clade, which is joined to Comamonadaceae clade.

Similar disagreements between two phylogenetic trees were revealed for Ralstonia genus too. Ralstonia is usually attributed to Ralstoniaceae on the base of the gltB sequences, but according to All-Species Living Tree [37] Ralstonia is joined to Cupriavidus and fell into Oxalobacteraceae clade.

Consequently, according to our results, the polymorphism of gltB gene sequences was high and allowed describing substantial diversity of the Burkholderiales order members, defined the main taxonomical groups represented by Burkholderiaceae (Burkholderia, Pandoraea, and Lautropia), Alcaligenaceae (Achromobacter), and Comamonadaceae (Variovorax), and revealed significant differences between Lautropia and the other Burkholderiaceae taxa.

In conclusion, we identified and characterized quite a wide range of the Burkholderiales order bacteria which are vital for the healthcare units at present in Russia. They 
have been represented by five genera: Burkholderia, Pandoraea, Lautropia (Burkholderiaceae), Achromobacter (Alcaligenaceae), and Variovorax (Comamonadaceae). The most abundant were $B c c$ and $A$. xylosoxidans with prevalence of transmissible ST709 and ST728 of Burkholderia cenocepacia and the first and second genotypes of A. xylosoxidans. Also not common and unusual bacteria like Pandoraea pnomenusa, Variovorax paradoxus, Lautropia mirabilis, and Ralstonia spp. began to appear in the hospitals and were registered in the group of the patients' samples. These observations confirm profound changes in the spectrum of the microorganisms, causing the healthcare-associated infections over the past few years that can be associated with emergence and dissemination of novel antibiotic resistance from the natural reservoir to the clinical setting. So we may conclude that pathogenic potential of the Burkholderiales is on the increase. Clarification of some questions on bacteria phylogeny and future genomic analysis of Burkholderiales species will provide deeper large-scale insights into the evolution of virulence mechanisms. The timely identification of the Burkholderiales order representatives by genotyping is important to limit bacterial spread and so to resolve some epidemiological problems.

\section{Abbreviations}

$\begin{array}{ll}\text { MLST: } & \text { Multilocus Sequence Typing } \\ \text { ST: } & \text { Sequence type } \\ \text { DLV: } & \text { Double locus variant } \\ \text { ML: } & \text { Maximum likelihood } \\ \text { NJ: } & \text { Neighbor-joining } \\ \text { CF: } & \text { Cystic fibrosis and cystic fibrosis patient } \\ \text { NON: } & \text { Non-CF patient } \\ \text { RF: } & \text { Russian Federation } \\ \text { Bcc: } & \text { Burkholderia cepacia complex } \\ \text { Ach: } & \text { Achromobacter xylosoxidans } \\ \text { Lau: } & \text { Lautropia mirabilis } \\ \text { Ral: } & \text { Ralstonia solanacearum/Ralstonia pickettii } \\ \text { Aci: } & \text { Acidovorax citrulli } \\ \text { Var: } & \text { Variovorax paradoxus } \\ \text { Bor: } & \text { Bordetella bronchiseptica/Bordetella pertussis } \\ \text { Pan: } & \text { Pandoraea pnomenusa } \\ \text { Pse: } & \text { Pseudomonas aeruginosa. }\end{array}$

\section{Conflict of Interests}

The authors declare that there is no conflict of interests regarding the publication of this paper.

\section{References}

[1] M. B. Mearns, G. H. Hunt, and R. Rushworth, "Bacterial flora of respiratory tract in patients with cystic fibrosis, 1950-71," Archives of Disease in Childhood, vol. 47, no. 256, pp. 902-907, 1972.

[2] R. Hobson, I. Gould, and J. Govan, "Burkholderia (Pseudomonas) cepacia as a cause of brain abscesses secondary to chronic suppurative otitis media," European Journal of Clinical
Microbiology and Infectious Diseases, vol. 14, no. 10, pp. 908-911, 1995.

[3] A. R. Hauser, M. Jain, M. Bar-Meir, and S. A. McColley, "Clinical significance of microbial infection and adaptation in cystic fibrosis," Clinical Microbiology Reviews, vol. 24, no. 1, pp. 29-70, 2011.

[4] E. Yabuuchi and I. Yano, "Achromobacter gen. nov. and Achromobacter xylosoxidans (ex Yabuuchi and Ohyama 1971) nom. rev," International Journal of Systematic and Evolutionary Microbiology, vol. 31, no. 4, pp. 477-478, 1981.

[5] "Validation list No. 45," International Journal of Systematic Bacteriology, vol. 43, pp. 398-399, 1993.

[6] P. Gerner-Smidt, H. Keiser-Nielsen, M. Dorsch et al., "Lautropia mirabilis gen. nov., sp. nov., a Gram-negative motile coccus with unusual morphology isolated from the human mouth," Microbiology, vol. 140, no. 7, pp. 1787-1797, 1994.

[7] "Validation of the publication of new names and new combinations previously effectively published outside the IJSB, List No. 57," International Journal of Systematic Bacteriology, vol. 46, no. 2, pp. 625-626, 1996.

[8] T. Coenye, E. Falsen, B. Hoste et al., "Description of Pandoraea gen. nov. with Pandoraea apista sp. nov., Pandoraea pulmonicola sp. nov., Pandoraea pnomenusa sp. nov., Pandoraea sputorum sp. nov. and Pandoraea norimbergensis comb. nov," International Journal of Systematic and Evolutionary Microbiology, vol. 50, no. 2, pp. 887-899, 2000.

[9] C. R. Woese, "Bacterial evolution," Microbiological Reviews, vol. 51, no. 2, pp. 221-271, 1987.

[10] C. R. Woese, W. G. Weisburg, B. J. Paster et al., "The phylogeny of purple bacteria: the beta subdivision," Systematic and Applied Microbiology, vol. 5, no. 3, pp. 327-336, 1984.

[11] C. R. Woese, W. G. Weisburg, and C. M. Hahn, "The phylogeny of purple bacteria: the gamma subdivision," Systematic and Applied Microbiology, vol. 6, no. 1, pp. 25-33, 1985.

[12] A. B. Ibarz Pavón and M. C. J. Maiden, "Multilocus sequence typing," Methods in Molecular Biology, vol. 551, pp. 129-140, 2009.

[13] O. L. Voronina, M. Y. Chernukha, I. A. Shaginyan et al., "Characterization of genotypes for Burkholderia cepacia complex strains isolated from patients in hospitals of the Russian federation," Molecular Genetics, Microbiology and Virology, vol. 28, no. 2, pp. 64-73, 2013.

[14] S. M. Naser, K. E. Hagen, M. Vancanneyt, I. Cleenwerch, J. Swings, and T. A. Tompkins, "Lactobacillus suntoryeus Cachat and Priest 2005 is a later synonym of Lactobacillus helveticus (Orla-Jensen 1919) Bergey et al. 1925 (Approved Lists 1980)," International Journal of Systematic and Evolutionary Microbiology, vol. 56, no. 2, pp. 355-360, 2006.

[15] L. L. Guan, K. E. Hagen, G. W. Tannock, D. R. Korver, G. M. Fasenko, and G. E. Allison, "Detection and identification of Lactobacillus species in crops of broilers of different ages by using PCR-denaturing gradient gel electrophoresis and amplified ribosomal DNA restriction analysis," Applied and Environmental Microbiology, vol. 69, no. 11, pp. 6750-6757, 2003.

[16] T. Spilker, A. Baldwin, A. Bumford, C. G. Dowson, E. Mahenthiralingam, and J. J. LiPuma, "Expanded multilocus sequence typing for Burkholderia species," Journal of Clinical Microbiology, vol. 47, no. 8, pp. 2607-2610, 2009.

[17] “The Main Site EMBL-EBI," European Bioinformatics Institute, 2014, http://www.ebi.ac.uk/Tools/msa/clustalw2.

[18] "The Main Site PubMLSR of the Faculty of Zoology, Oxford University, Great Britain,” 2014, http://pubmlst.org. 
[19] K. Tamura, J. Dudley, M. Nei, and S. Kumar, "MEGA4: molecular evolutionary genetics analysis (MEGA) software version 4.0," Molecular Biology and Evolution, vol. 24, no. 8, pp. 15961599, 2007.

[20] K. Tamura, G. Stecher, D. Peterson, A. Filipski, and S. Kumar, "MEGA6: molecular evolutionary genetics analysis version 6.0," Molecular Biology and Evolution, vol. 30, no. 12, pp. 2725-2729, 2013.

[21] D. H. Huson, "SplitsTree: analyzing and visualizing evolutionary data," Bioinformatics, vol. 14, no. 1, pp. 68-73, 1998.

[22] N. Saitou and M. Nei, "The neighbor-joining method: a new method for reconstructing phylogenetic trees," Molecular Biology and Evolution, vol. 4, no. 4, pp. 406-425, 1987.

[23] M. Nei and S. Kumar, Molecular Evolution and Phylogenetics, Oxford University Press, New York, NY, USA, 2000.

[24] K. Tamura, "Estimation of the number of nucleotide substitutions when there are strong transition- transversion and $\mathrm{G}+$ C-content biases," Molecular Biology and Evolution, vol. 9, no. 4, pp. 678-687, 1992.

[25] D. Posada and K. A. Crandall, "MODELTEST: testing the model of DNA substitution,” Bioinformatics, vol. 14, no. 9, pp. 817-818, 1998.

[26] K. A. Kormas, "Interpreting diversity of Proteobacteria based on 16S rRNA gene copy number," in Proteobacteria: Phylogeny, Metabolic Diversity and Ecological Effects, M. L. Sezenna, Ed., pp. 73-89, Nova Publishers, Hauppauge, NY, USA, 2011.

[27] NCBI, Genome List, http://www.ncbi.nlm.nih.gov/genome/ browse/.

[28] H.-C. Yang, W.-T. Im, K. K. Kim, D.-S. An, and S.-T. Lee, "Burkholderia terrae sp. nov., isolated from a forest soil," International Journal of Systematic and Evolutionary Microbiology, vol. 56, part 2, pp. 453-457, 2006.

[29] J. P. McCutcheon and N. A. Moran, "Functional convergence in reduced genomes of bacterial symbionts spanning $200 \mathrm{My}$ of evolution," Genome Biology and Evolution, vol. 2, no. 1, pp. 708718, 2010.

[30] P. Drevinek and E. Mahenthiralingam, "Burkholderia cenocepacia in cystic fibrosis: epidemiology and molecular mechanisms of virulence," Clinical Microbiology and Infection, vol. 16, no. 7, pp. 821-830, 2010.

[31] J. P. Euzéby, "The List of Prokaryotic names with Standing in Nomenclature," http://www.bacterio.net.

[32] P. Vandamme, E. R. B. Moore, M. Cnockaert et al., "Classification of Achromobacter genogroups 2, 5, 7 and 14 as Achromobacter insuavis sp. nov., Achromobacter aegrifaciens sp. nov., Achromobacter anxifer sp. nov. and Achromobacter dolens sp. nov., respectively," Systematic and Applied Microbiology, vol. 36, no. 7, pp. 474-482, 2013.

[33] The phylogentic tree for the order Burkholderiales, http:// patricbrc.org/portal/portal/patric/Phylogeny?cType=taxon\& cId $=47671$.

[34] A. R. Wattam, D. Abraham, O. Dalay et al., "PATRIC, the bacterial bioinformatics database and analysis resource," Nucleic Acids Research, vol. 42, no. 1, pp. D581-D591, 2014.

[35] F. Nagai, M. Morotomi, H. Sakon, and R. Tanaka, "Parasutterella excrementihominis gen. nov., sp. nov., a member of the family Alcaligenaceae isolated from human faeces," International Journal of Systematic and Evolutionary Microbiology, vol. 59, no. 7, pp. 1793-1797, 2009.

[36] H. M. Wexler, D. Reeves, P. H. Summanen et al., "Sutterella wadsworthensis gen. nov., sp. nov., bile-resistant microaerophilic Campylobacter gracilis-like clinical isolates," International Journal of Systematic Bacteriology, vol. 46, no. 1, pp. 252-258, 1996.

[37] “The All-Species Living Tree (Release LTPs115)," March 2014, http://www.arb-silva.de/fileadmin/silva_databases/living_tree/ LTP_release_115/LTPs115_SSU_tree.pdf. 

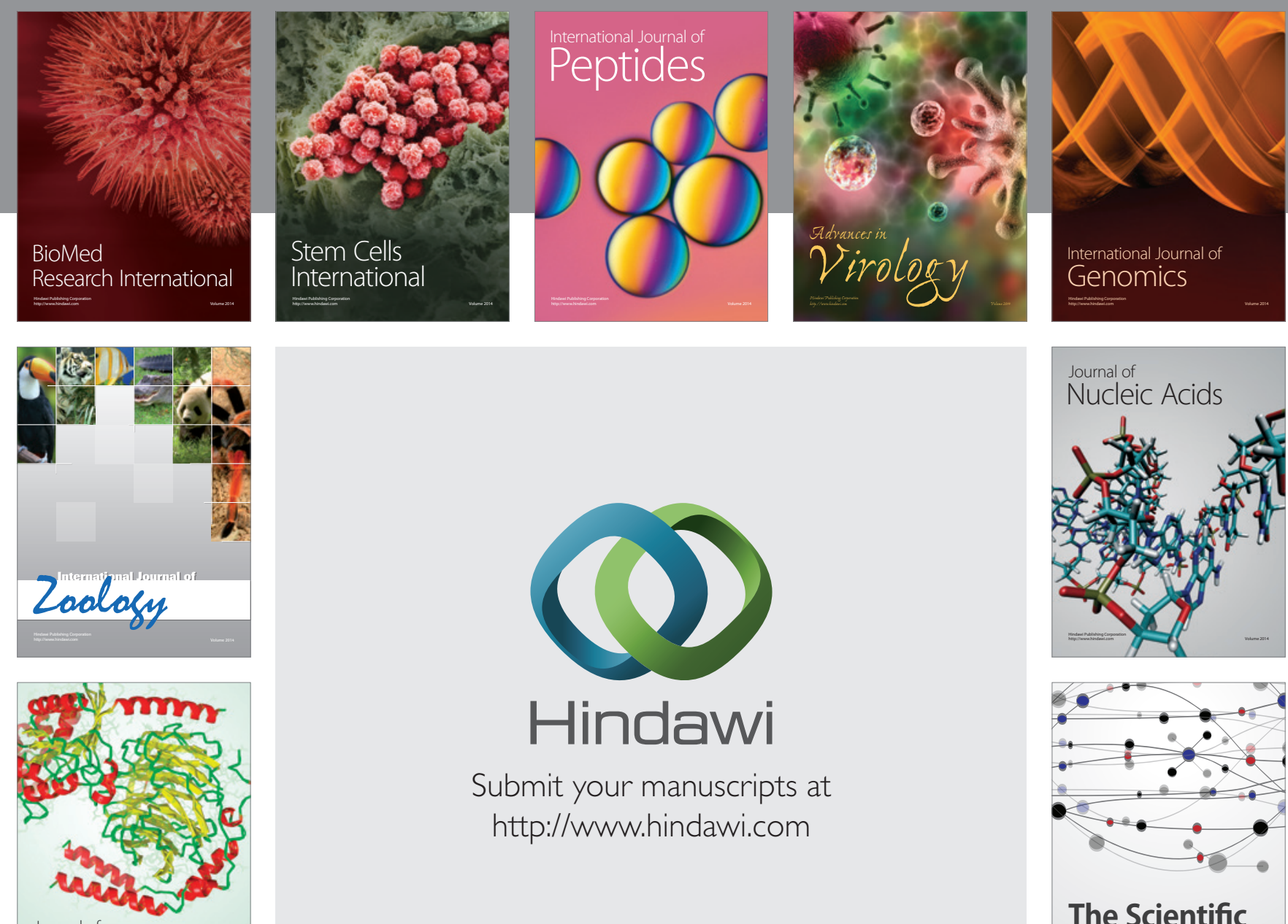

Submit your manuscripts at

http://www.hindawi.com

Journal of
Signal Transduction
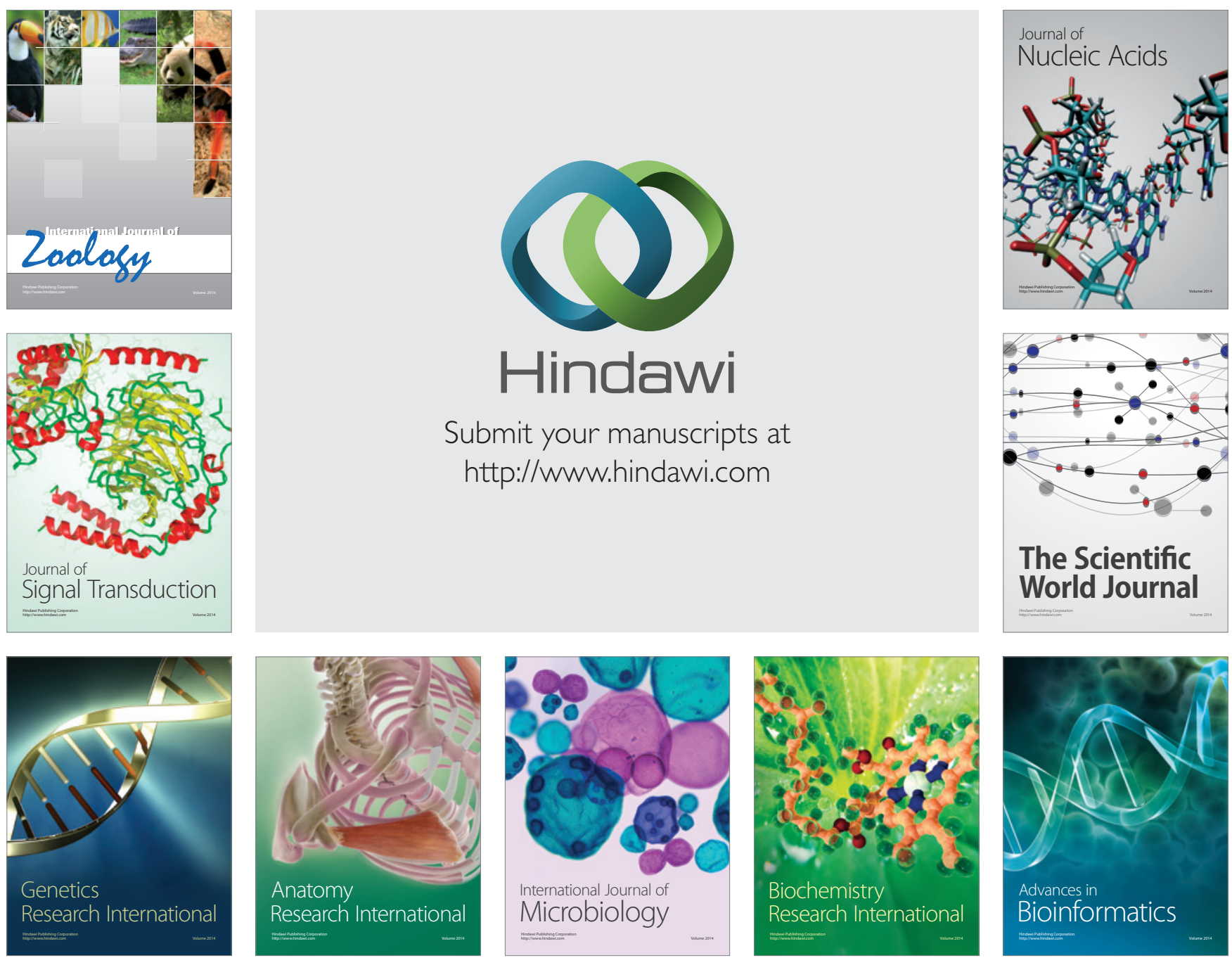

The Scientific World Journal
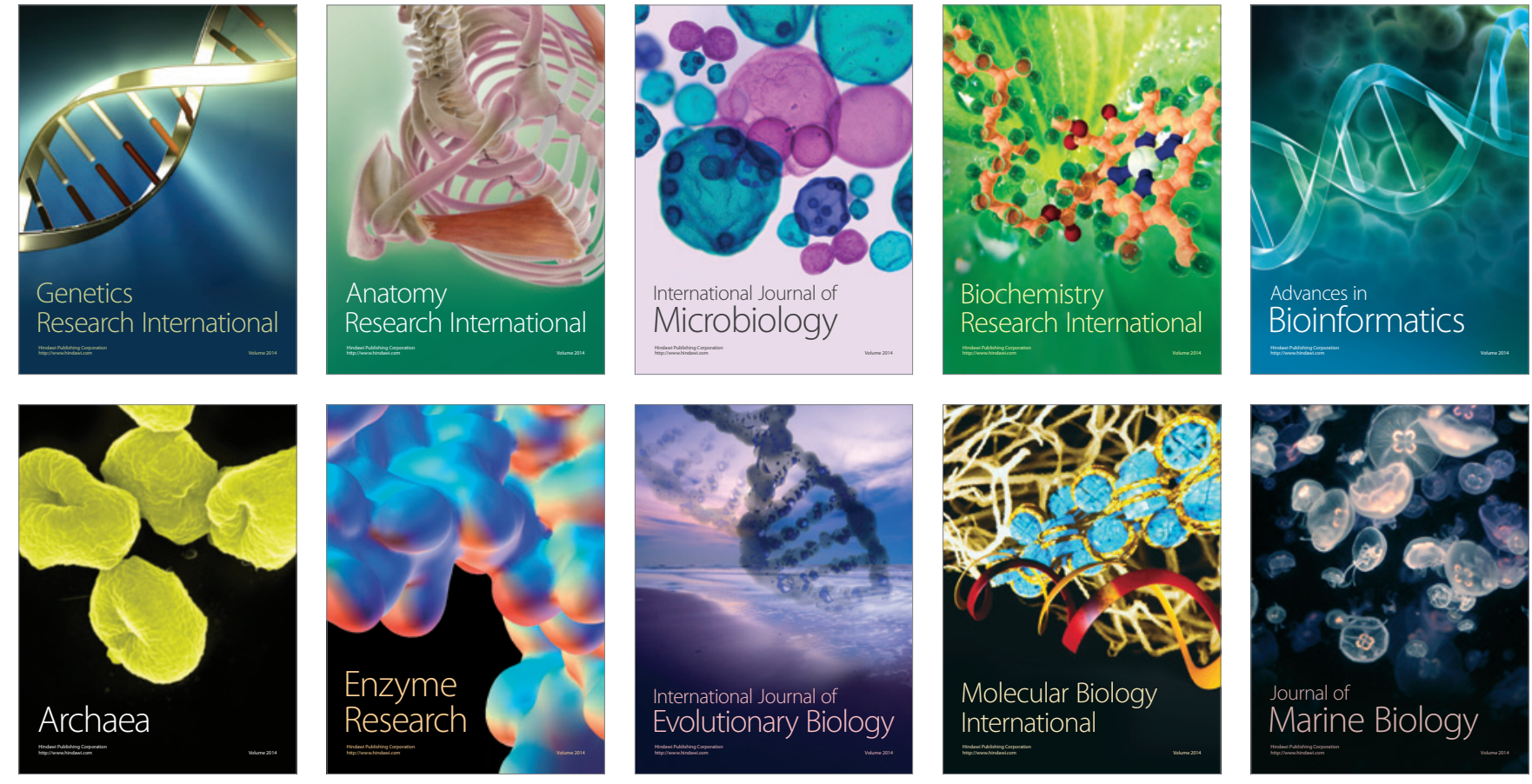